\title{
Targeted Protein Detection using an All-In-One Mass Spectrometry Cartridge
}

\author{
Chengsen Zhang, ${ }^{\dagger}$ Trevor Glaros, ${ }^{\ddagger}$ and Nicholas E Manicke*,† \\ ${ }^{\dagger}$ Department of Chemistry and Chemical Biology, Indiana University-Purdue University Indianapolis, Indianapolis, IN \\ 46202, USA \\ ${ }^{\ddagger}$ BioSciences Division, BioDefense Branch, US Army Edgewood Chemical Biological Center, Aberdeen Proving Ground, \\ MD 21010, USA
}

Supporting Information Placeholder

\begin{abstract}
We developed a simple 3D printed cartridge for mass spectrometry (MS) targeted detection of plasma proteins, including post-translational modifications (PTMS). The cartridge uses an integrated antibody enrichment column to preconcentration the protein target as well as a novel built-in substrate to ionize the protein targets for MS detection. We show several examples of using this cartridge to perform rapid detection of clinically significant proteoforms from plasma samples.
\end{abstract}

Protein molecules and targets are measured in clinical diagnostic laboratories as tumor markers, acute or chronic disease markers, risk or prognostic markers, and hormones. Immunoassays have been successfully used for decades to detect protein analytes in clinical laboratories, but there remain limitations of this approach. Monoclonal antibodies are expensive to develop and manufacture. Moreover, there are well recognized analytical limitations; for example, most immunoassays do not distinguish between the various proteoforms and variants of a protein present in clinical samples. ${ }^{1}$

Targeted enrichment of proteins followed by MS detection is a widely used approach to detect different proteoforms. Enrichment is generally performed using antibodies because of their ability to selectively enrich target proteins from protein rich matrices such as plasma. Mass spectrometric immunoassay (MSIA) uses antibody coated beads packed into a pipette tip ${ }^{2}$ followed by detection of intact proteins by MALDI-MS. Another approach is antibody modification of the MALDI target. $^{3-5}$ In SISCAPA (stable isotope standards and capture by antipeptide antibodies) and immuno-MALDI, enrichment is performed at the peptide level after digestion. ${ }^{6-7}$ Peptide level enrichment gives better quantitation and improved sensitivity. However, limited sequence coverage compli- cates detection of PTMs and sequence variants.

Development of a simple MS assay capable of resolving proteoforms would have a significant impact on protein detection, including clinical testing. We previously reported simple, single-use MS cartridges for the analysis of drugs which integrated sample preparation, sample storage as a dried spot, and analyte ionization via paper spray, in which paper is used as an inexpensive solid support for extraction, fluid transport, and electrospray ionization. ${ }^{8-9}$ Here, we describe the development of a mass spectrometry cartridge designed for targeted detection of proteins from complex biofluids such as plasma. Selective enrichment of protein targets is performed oncartridge passively by capillary action and gravity with no pumping. Detection of the intact protein targets then proceeds via ionization of the protein target using a built-in spray substrate consisting of carbon-nanotube (CNT) coated porous polyethylene. The distinguishing feature of this approach is its simplicity and low cost, in effect a combination of inexpensive lateral flow assays with mass spectrometry. Three applications of this cartridge are described for clinically significant problems: 1) identification of T45S variant of apolipoprotein $\mathrm{c} 1$, a polymorphism associated with body mass index and obesity, 2) relative quantitation of hemoglobin ( $\mathrm{Hb})$ A1C, a marker of diabetes, and 3) identification of sequence variants of transthyretin, which are markers of transthyretin-related hereditary amyloidosis.

A 3D printed cartridge (Figure 1) was developed to perform the entire analyte preconcentration and ionization process. An absorbent waste pad in the base of the cartridge draws the sample through the antibody column, which consisted of a plastic tube packed with glass fiber membranes coated with latex bead-antibodies conjugates. All parts were assembled together, and the column holder was slid along the groove between lid and base to switch the position of the antibody column from above 
the waste pad, where the sample addition and washing steps occurred, to above the spray substrate where ionization by substrate supported electrospray ionization occurred. MS detection was performed on a quadrupole-orbitrap mass spectrometer in full scan mode.

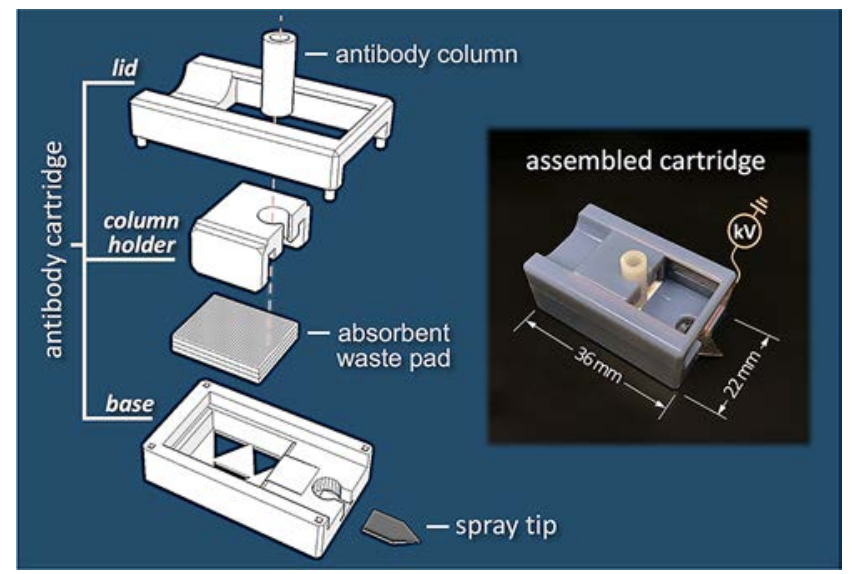

Figure 1. Schematic diagram and photograph of the antibody cartridge. The inserted photograph shows the assembled cartridge in the sample loading/wash. The antibody column extracted target protein from the plasma and a CNT-coated porous polyethylene spray tip was used for substrate supported electrospray.

A number of reports describe the use of paper spray MS for the detection of drugs and other small molecules from blood. ${ }^{10-11}$ The detection efficiency of proteins by paper spray is relatively poor, however. A paper spray based immunoassay was reported in which a charged probe released from the bound antibody was detected by MS. ${ }^{12}$ Although this approach has better sensitivity because a small molecule is used for detection, the selectivity is ultimately limited by the antibody. Detection of the probe cannot give any information on the antigen such as PTMs. Improved protein ionization has been reported using paper coated with $\mathrm{CNT}^{13}$ as well as size exclusion membranes. ${ }^{14}$ Carbon nanotube coated paper has also been utilized for low voltage paper spray. ${ }^{15} \mathrm{We}$ investigated CNT treated paper and as well as a novel substrate, CNT treated porous polyethylene (PE). Photographs and scanning electron microscope images of the spray substrates are shown in Figure S1. Detection limits of the three proteins were improved by coating or dispersing chromatography paper with single-walled CNT (Table S1). The CNT-coated PE even further improved protein detection; CNT-coated PE detection limits were between 10 and 100 times lower compared to the best obtained using CNT treated paper. Detection limits of the protein standards were improved by a factor of 501000 compared to typical paper spray. Mass spectra obtained using paper and CNT-coated PE for three standard protein solutions are shown in Figure 2.

The Taylor cone generated from the CNT-coated PE tip was significantly smaller compared to paper (Figure
3). The size of the Taylor cone correlates with droplet size, and smaller electrospray droplets correlate with better ionization efficiency. ${ }^{16}$ The improved protein detection from the CNT-coated PE substrate therefore may arise from balancing the surface energy of the substrate with that of the solvent to generate smaller droplets and more efficient ionization. The substrate must be sufficiently wettable to allow wicking by a polar solvent, necessary to drive fluid and analyte transport, but not so wettable that the Taylor cone is large. The CNT-PE substrate was also found to retain less protein than paper, but the difference was small compared to the improvement in detection limits (supplementary material). (a)

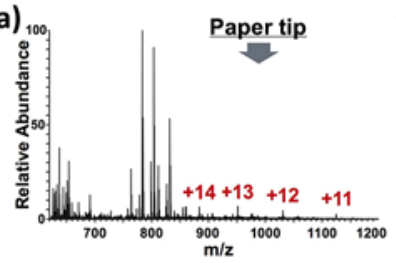

(b)

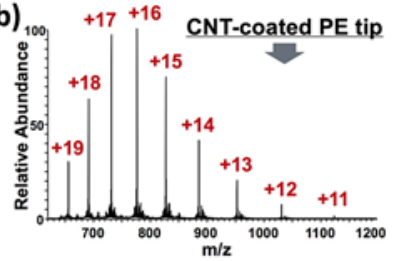

Figure 2. Full MS spectra obtained by ionizing $10 \mu \mathrm{g} / \mathrm{mL}$ cytochrome c using paper spray (a) and the CNT-coated PE substrate (b). Spectra for two other proteins are in supplementary figure $\mathrm{S} 2$.

To achieve selective and sensitive detection of target proteins from biofluids, we utilized an antibody column integrated into the cartridge. In this method, protein preconcentration was performed using a membrane containing latex bead-antibody conjugates. The plasma sample was added to the antibody column where it flowed through by a combination of capillary action and gravity. Excess sample was absorbed into a built-in waste pad in the bottom cartridge, while the target protein was retained on the latex bead conjugates. A washing step was performed by subsequently adding water to the preconcentration column, where it wicked into the waste pad. The protein analyte was then detected by sliding the antibody column to the elution position above the spray substrate and adding 1:1 methanol:water with $2 \%$ acetic acid, which acted as both the extraction solution and the spray solvent. Over $90 \%$ of captured protein was eluted in the case of cytochrome C (supplemental material).

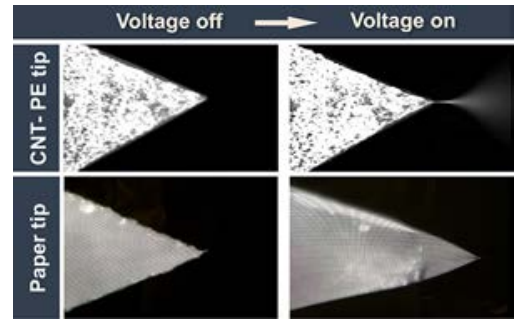

Figure 3. Taylor cone generated from CNT-PE and paper. The diameter of the Taylor cone generated from the sharp point of CNT-PE was much smaller compared with paper. CNT-coated paper is pictured in supplementary figure S3.

We used polyclonal antibodies as opposed to mono- 
clonal antibodies. While monoclonal antibodies are generally preferred in immunoassays because of their higher selectivity, polyclonal antibodies have a number of advantages for this application. Polyclonal antibodies have higher affinity compared to monoclonal antibodies, they bind to multiple different epitopes which minimizes the risk that a particular proteoform or variant will not bind, and they are cheaper than monoclonal antibodies. The lower selectivity of polyclonal antibodies is not a factor in this application because of the use of MS detection. This approach does necessitate that antibodies against the target protein be available. Antibodies are also the most expensive component of the cartridge. However, use of antibodies in this cartridge are still significantly cheaper with faster development times than traditional immunoassays. Because MS detection is used, high selectivity monoclonal antibodies are not required, which decreases the cost and time associated with antibody generation.

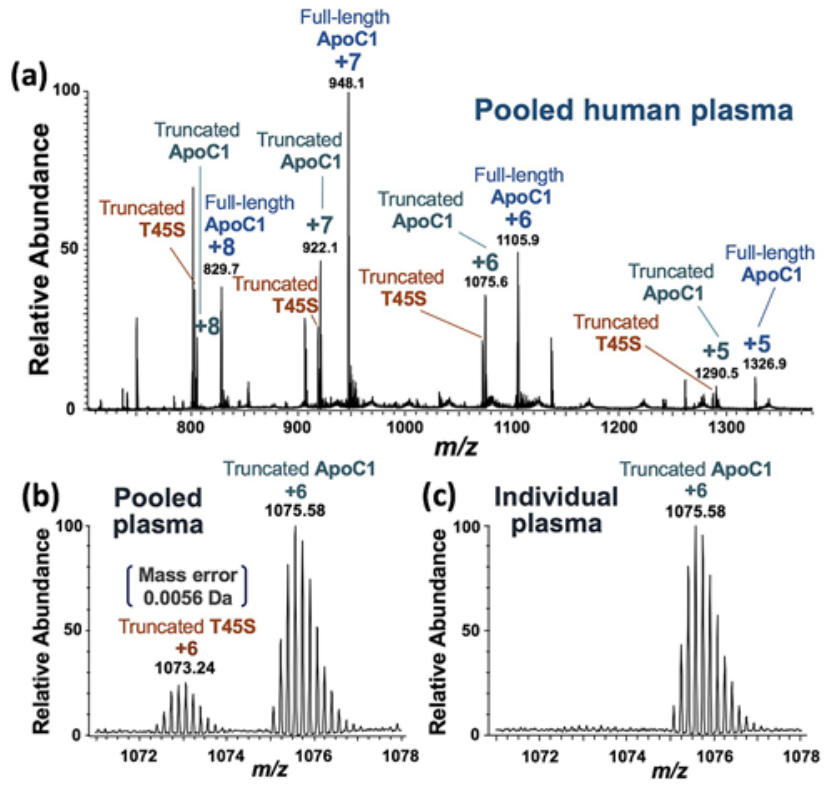

Figure 4. The MS protein detection cartridge analysis of human plasma apolipoprotein c1 (ApoC1). (a) full MS spectrum obtained from the pooled human plasma sample showing the ApoC1 full-length, truncated, and truncated T45S variant. Narrower MS scan range shows the truncated ApoC1 +6 ions obtained from (b) pooled human plasma and (c) individual donated plasma.

We investigated three clinically significant applications of the MS protein detection cartridge. Apolipoprotein C1 (ApoC1) T45S variant is a naturally occurring amino acid polymorphism that has been associated with elevated body mass index and diabetes. ${ }^{17}$ As shown in Figure 4a, three ApoC1 species were identified in the MS spectrum obtained from a pooled human plasma sample, including full-length ApoC1 (6.6 kDa), truncated ApoC1(minus amino-terminal Thr-Pro, $6.4 \mathrm{kDa}$ ), and truncated T45S variant $(\Delta \mathrm{m}=-14.03 \mathrm{Da})$. Detection of the T45S variant in the pooled plasma sample indicated that some of plasma came from individuals with the ApoC1 S45 allele (figure 4b). The protein cartridge was also used to analyze plasma from a single donor; the mass spectrum indicates that the individual donor did not have this allele (figure 4c). In comparison with calculated accurate masses, mass error for detected T45S variant was $0.0056 \mathrm{Da}$. These results indicate the method has good potential for rapid detection of the ApoC1 T45S variant.
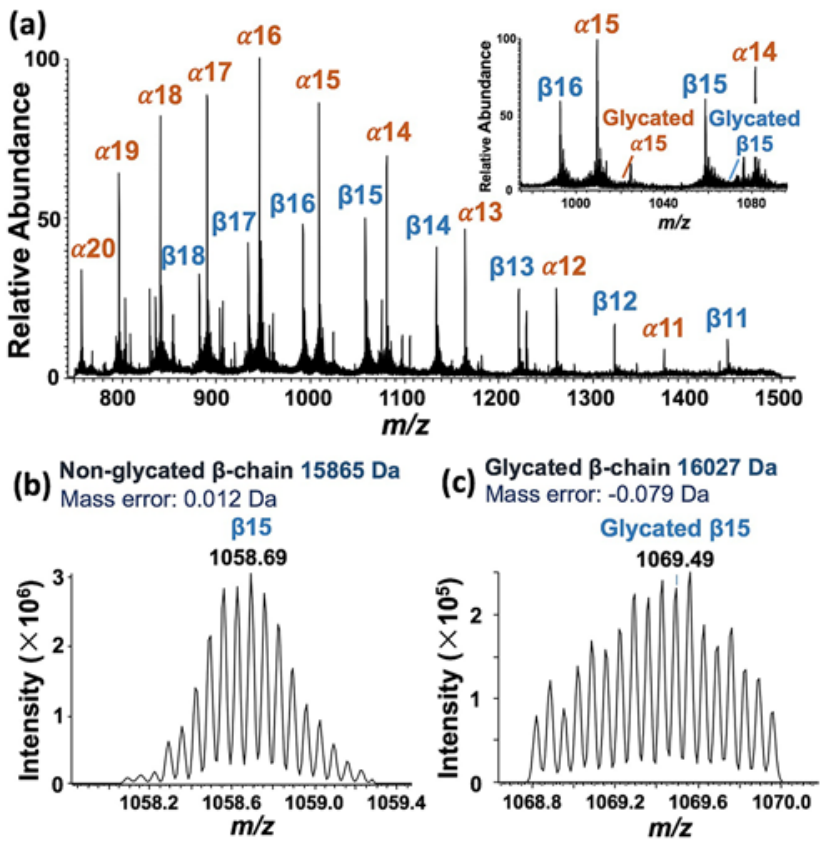

Figure 5. Antibody cartridge analysis of human plasma hemoglobin (Hb). (a) full MS spectrum shows the $\mathrm{Hb} \alpha-/ \beta$ chain ions with charge state from +11 to +20 . SIM spectra $(\mathrm{m} / \mathrm{z}$ scan window $1.2 \mathrm{Da})$ show the relative intensities of non-glycated (b) and glycated (c) Hb.

Glycated hemoglobin is a stable minor $\mathrm{Hb}$ variant formed in vivo by the non-enzymatic, covalent attachment of glucose. Relative quantitation of a particular $\mathrm{Hb}$ fraction, $\mathrm{HbA}_{1 \mathrm{c}}$, has been used as a marker of glycemia control in diabetes for decades. ${ }^{18}$ As shown in Figure 5, several hemoglobin species were identified using the protein detection cartridge, such as nonglycated $\alpha$ - $/ \beta$ chains (Figure $5 \mathrm{a}$ ), and glycated $\alpha$-/ $\beta$-chains (Figure $5 \mathrm{a}$ insert spectrum). The $\mathrm{Hb}$ and glycated $\mathrm{Hb}$ were monitored using single ion monitoring (SIM) of the +15 charge state as shown in figure $5 \mathrm{~b}$ and $5 \mathrm{c}$. The mass error for the detected non-glycated $\mathrm{Hb} \beta$-chain and glycated $\mathrm{Hb} \beta$-chain were $0.012 \mathrm{Da}$ and $-0.079 \mathrm{Da}$, respectively. The relative intensity of glycated $\mathrm{Hb}$ was $\sim 7 \%$ compared to unglycated $\mathrm{Hb}$, which is similar to the expected proportion. ${ }^{19-20}$ The results indicate the method has potential for relative quantitation of $\mathrm{Hb}$ and $\mathrm{HbA}_{1 \mathrm{c}}$.

Transthyretin (TTR) is a $55 \mathrm{kDa}$ homotetramer protein that transports thyroxine and retinol in blood and cerebrospinal fluid. Misfolding and aggregation of wild-type (wt) and mutant transthyretin protein (TTR) is the cause 
of transthyretin amyloidosis (ATTR), a severe and fatal disease characterized by abnormal deposits of TTR amyloids. Mass spectrometry is used to screen for ATTR. ${ }^{20}$ The antibody cartridge was successfully used to detect wt TTR as well as various TTR mutants from human plasma samples. Supplementary Figure S4 shows the mass spectrum obtained for the +10 charge state of $\mathrm{wt}$ TTR. The unmodified TTR was a relatively minor component; the cysteine, glutathione, and sulfonate PTMs were all more intense. To reduce the complexity of the mass spectrum, we spiked the reducing agent Tris(2carboxyethyl)phosphine hydrochloride (TCEP-HCl). This procedure, performed on-cartridge in the extraction/spray solvent without a separate step, succeeding in eliminating the S-cysteine and S-glutathione modifications (Figure S4), although the S-sulfonation remained. Four different TTR mutants in six ATTR clinical samples were clearly identified and differentiated from wt TTR. Figure 6 shows the charge-state deconvoluted mass spectra obtained from a wt sample and a representative ATTR sample. The spectrum from the ATTR sample indicates the patient is heterozygous for wild type TTR and a TTR variant with a $\Delta \mathrm{m}$ of $32.06 \mathrm{Da}$. This mass shift is consistent with substitution of valine30 for methionine, the most common mutation found in TTR associated neuropathy. ${ }^{22}$
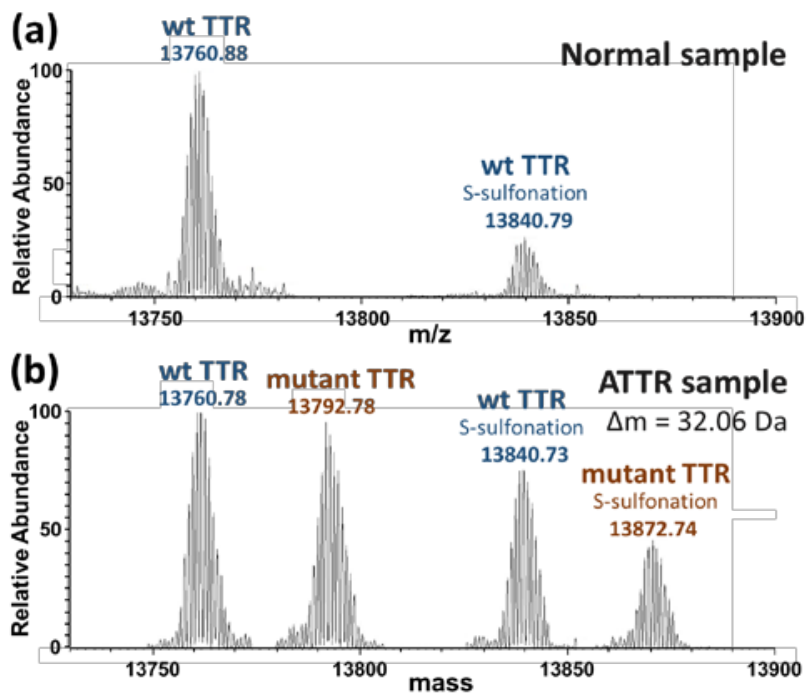

Figure 6. The charge-state deconvoluted MS spectra obtained by antibody cartridge analysis of human plasma from a wt sample (a) and a ATTR plasma sample (b). Only wild type TTR, including free thiol wt-TTR and Ssulfonation wt-TTR, can be detected from normal sample, while a mutant TTR with $\Delta \mathrm{m}=32.0$ Da was identified from the ATTR sample.

In summary, we describe a simple, all-in-one 3D printed cartridge which enables targeted protein detection from plasma samples by mass spectrometry. The unique feature of this device is that both protein enrichment as well as ionization occur from one device. Because of the simplicity of the analytical procedure, this cartridge better enables MS-based detection of protein targets, which is important in clinical diagnostics and other fields. While MS is expensive relative to traditional immunoassay detection methods, mass spectrometry is better suited for distinguishing post-translational modifications, sequence variants, and other proteoforms.

\section{ASSOCIATED CONTENT}

\section{Supporting Information.}

The Supporting Information is available free of charge on the ACS Publications website.

Materials, full experimental details, supplemental figures S1-S4 and supplemental table S1 (PDF)

\section{AUTHOR INFORMATION}

\section{Corresponding Author}

*E-mail: nmanicke@iupui.edu

\section{Notes}

The authors declare no competing financial interest.

\section{ACKNOWLEDGMENT}

The authors acknowledge Thermo Scientific for funding and other supporting. We also thank Merrill Benson MD, IU School of Medicine, for supplying the ATTR samples.

\section{REFERENCES}

(1) Anderson, N. L. Clin. Chem. 2010, 56, 177.

(2) Nelson, R. W.; Krone, J. R.; Bieber, A. L.; Williams, P. Anal. Chem. 1995, 67, 1153

(3) Liang, X. L.; Lubman, D. M.; Rossi, D. T.; Nordblom, G. D.; Barksdale, C. M. Anal. Chem. 1998, 70, 498.

(4) Min, D. H.; Su, J.; Mrksich, M. Angew. Chem., Int. Ed. 2004, 43, 5973.

(5) Pompach, P.; Novakova, J.; Kavan, D.; Benada, O.; Ruzicka, V.; Volny, M.; Novak, P. Clin. Chem. 2016, 62, 270.

(6) Anderson, N. L.; Anderson, N. G.; Haines, L. R.; Hardie, D. B.; Olafson, R. W.; Pearson, T. W. J. Proteome Res. 2004, 3, 235.

(7) Warren, E. N.; Elms, P. J.; Parker, C. E.; Borchers, C. H. Anal. Chem. 2004, 76, 4082.

(8) Zhang, C.; Manicke, N. E. Anal. Chem. 2015, 87, 6212.

(9) Bills, B. J.; Manicke, N. E. Clin. Mass Spectrom. 2016, 2, 18.

(10) Wang, H.; Liu, J.; Cooks, R. G.; Ouyang, Z. Angew. Chem., Int. Ed. 2010, 49, 877. 589.

(11) Manicke, N. E.; Bills, B. J.; Zhang, C. Bioanalysis 2016, 8,

(12) Chen, S.; Wan, Q.; Badu-Tawiah, A. K. J. Am. Chem. Soc 2016, 138, 6356.

(13) Han, F.; Yang, Y.; Ouyang, J.; Na, N. Analyst 2015, 140, 710.

(14) Zhang, M.; Lin, F. K.; Xu, J. G.; Xu, W. Anal. Chem. 2015, 87, 3123.

(15) Narayanan, R.; Sarkar, D.; Cooks, R. G.; Pradeep, T. Angew. Chem., Int. Ed. 2014, 53, 5936.

(16) Juraschek, R.; Dulcks, T.; Karas, M. J. Am. Soc. Mass Spectrom. 1999, 10, 300.

(17) Kasthuri, R. S.; McMillan, K. R.; Flood-Urdangarin, C.; Harvey, S. B.; Wilson-Grady, J. T.; Nelsestuen, G. L. Int. J. Obes. (Lond) 2007, 31, 1334.

(18) Gillery, P. Clin. Chem. Lab. Med. 2013, 51, 65.

(19) Weykamp, C. Ann. Lab. Med. 2013, 33, 393.

(20) Lim, A.; Prokaeva, T.; McComb, M. E.; O'Connor, P. B.; Théberge, R.; Connors, L. H.; Skinner, M.; Costello, C. E. Anal. Chem. 2002, 74, 741.

(21) Saraiva, M. J. M. J. Peripher. Nerv. Syst. 1996, 1, 179. 
For Table of Contents Only

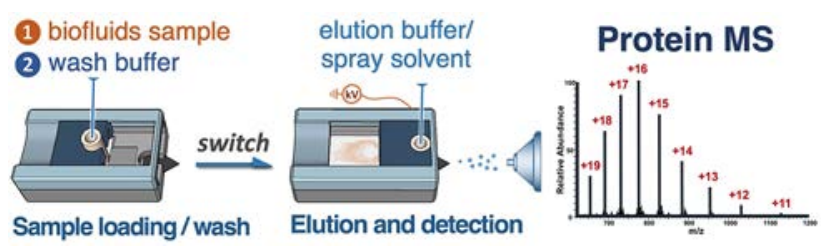




\title{
Supporting Information
}

\section{Targeted Protein Detection using an All-In-One Mass Spectrometry Cartridge}

\author{
Chengsen Zhang, ${ }^{\dagger}$ Trevor Glaros, ${ }^{\ddagger}$ and Nicholas E Manicke*,† \\ ${ }^{\dagger}$ Department of Chemistry and Chemical Biology, Indiana University-Purdue University Indianapolis, Indianapolis, \\ IN 46202, USA \\ *BioSciences Division, BioDefense Branch, US Army Edgewood Chemical Biological Center, Aberdeen Proving \\ Ground, MD 21010, USA
}

\section{Contents}

S1. Chemicals and materials

S2. Preparation of spray substrates

S3. Photograph and SEM image of spray substrates

S4. Full MS spectra of two proteins obtained using paper spray and CNT-coated PE substrate

S5. Detection limits of standard protein solution obtained by different spray substrates

S6. Preparation of antibody coated membrane

S7. Sample preparation

S8. Experimental methods

S9. Taylor cone formed from CNT-coated paper

S10. Reduction of TTR posttranslational modifications

S11. Protein retention by the spray substrates

S12. Recovery of captured protein 


\section{S1. Chemicals and materials}

Three human plasma samples were tested, including individual donated plasma, pooled human plasma, and deidentified plasma samples from patients with transthyretin amyloidosis (ATTR samples). Anti-hemoglobin polyclonal antibody was purchased from Thermo Fisher Scientific (Rockford, IL, USA). Anti-transthyretin polyclonal antibody was purchased from LifeSpan BioSciences, Inc. (Seattle, WA, USA). Carboxyl latex beads $(1.4 \mu \mathrm{m})$ was purchased from Molecular Probes, Inc. (Eugene, OR, USA). 1-(3-Dimethylaminopropyl)3-ethylcarbodiimide hydrochloride (EDAC hydrochloride) was purchased from Acros Organics (Fair Lawn, NJ, USA). Methanol and acetic acid was purchased from Fisher Scientific Co. (Fair Lawn, NJ, USA). Millipore glass-fiber membrane filters, single-walled carbon nanotubes $(0.7-1.3 \mathrm{~nm})$, and all other proteins and chemicals were purchased from Sigma-Aldrich (St. Louis, MO, USA). Amicon Ultra $0.5 \mathrm{~mL}$ centrifugal filter was purchased from Merck Millipore Ltd. (Tullagreen, Cork, Ireland). The antibody cartridge was 3D printed using a Stratasys Objet 30 pro using VeroBlue photopolymer (Eden Prairie, MN, U.S.A). The antibody column was cut out of Delrin plastic tube (McMaster-Carr) (Elmuhurst, IL, U.S.A.). Porous polyethylene was purchased from Interstate Specialty Products (Sutton, MA, USA). 


\section{S2. Preparation of spray substrates}

Pretreatment of carbon nanotubes. The single-walled carbon nanotube powder was washed by immersion in methanol: water 1:1(v: v) and sonication for 15 minutes (3X), and followed by immersion in pure methanol and sonication for 15 minutes (3X), and then store in pure methanol as a slurry with a concentration about 10 $\mathrm{mg} / \mathrm{mL}$.

Preparation of porous polyethylene (PE). The PE was cut into rectangles $(2.0 \mathrm{~cm} \mathrm{X} 0.5 \mathrm{~cm})$ and then washed by immersion in pure methanol and vortexing for 1 minute (3X) and followed by immersion in 1:1 methanol: water and vortexing for a minute (3X). Washed PE was than allow to dry before applying CNT.

Preparation of CNT-dispersed spray substrates. The spray substrates, chromatography paper and porous polyethylene, were immersed in the CNT slurry and sonicated for $30 \mathrm{~min}$. CNT-dispersed spray substrates were allow to dry and cut into pentagon shape with a sharp tip, as shown in Figure S1.

Preparation of CNT-coated spray substrates. The paper substrate and washed thin piece of PE were placed on an absorbent pad, and the CNT slurry $(10 \mathrm{mg} / \mathrm{mL}$ in methanol) was pipetted onto the upper surface of spray substrates. After the solvent pass though the spray substrate to the absorbent pad, most of the CNT stay on the surface of spray substrates. CNT-coated paper substrate was ready to use after drying and cut into pentagon shape with a sharp tip. CNT-coated PE need more treatment after drying, as follows: the upper surface was polished with a Kim wipe to remove the excess CNT and form a thin CNT-coated layer on the PE substrate, and then washed again as described in the preparation of porous polyethylene. The CNT coated PE substrate was allowed to dry, then cut into spray tips. To form the spray tip, one side of the rectangle (short side) was cut evenly to a fine triangular point. The CNT-PE tips could be re-used after washing. 


\section{S3. Photograph and SEM image of spray substrates}

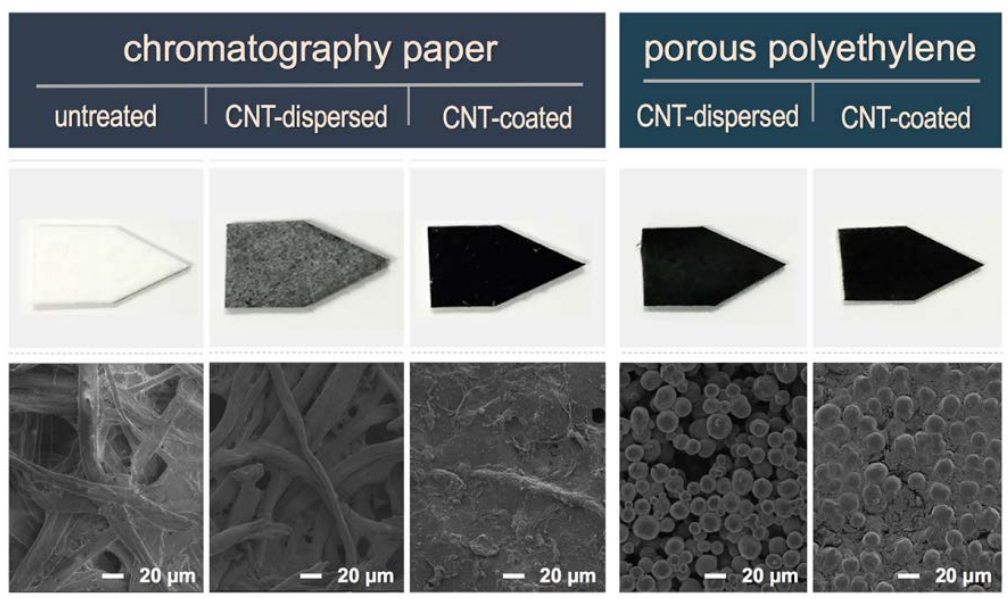

Figure S1. Photograph and SEM image of different spray substrates. Spray substrate from left to right: untreated chromatography paper, CTN-dispersed chromatography paper, CNT-coated chromatography paper, CTN-dispersed porous polyethylene, and CTN-coated porous polyethylene. 
S4. Full MS spectra of two proteins obtained using paper spray and CNT-coated PE substrate

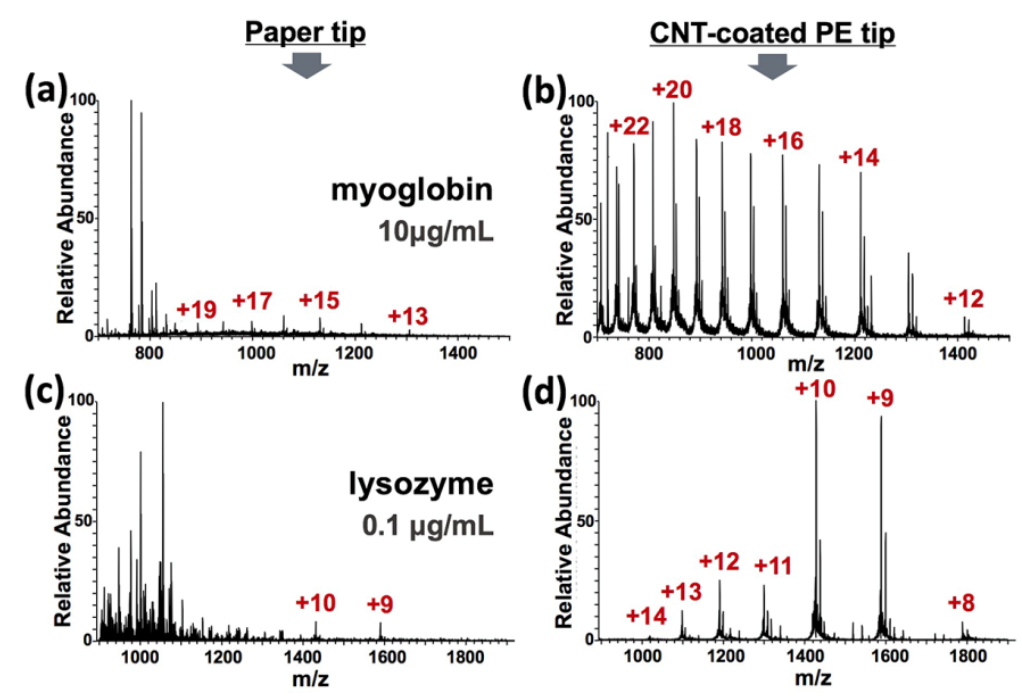

Figure S2. Full MS spectra obtained by ionizing $10 \mu \mathrm{g} / \mathrm{mL}$ myoglobin and $0.1 \mu \mathrm{g} / \mathrm{mL}$ lysozyme using paper spray (a, c) and the CNT-coated PE substrate (b, d). 


\section{S5. Detection limits of standard protein solution obtained by different spray substrates}

Table S1 lists the detection limits of three standard protein solution obtained by using the five different spray substrates shown in Figure S1. Detection limits were determined experimentally by analysis of serial dilutions of protein standards $(\mathrm{S} / \mathrm{N}>3)$.

Table S1. Limits of detection of proteins obtained by paper and PE spray substrates.

\begin{tabular}{clccc}
\hline \multirow{2}{*}{ Spray substrates } & \multicolumn{3}{c}{ Detection limits $(\mu \mathrm{g} / \mathrm{mL})$} \\
& & Cytochrome c & Myoglobin & Lysozyme \\
\hline \multirow{2}{*}{ Paper } & Untreated & 3 & 5 & 100 \\
& CNT-dispersed & 1 & 0.8 & 10 \\
& CNT-coated & 0.5 & 1 & 100 \\
\hline \multirow{2}{*}{ PE } & CNT-dispersed & 0.05 & 0.2 & 0.3 \\
& CNT-coated & 0.01 & 0.1 & 0.1 \\
\hline
\end{tabular}




\section{S6. Preparation of antibody coated membrane}

To prepare the antibody coated membrane, the antibodies were coated onto carboxyl latex beads first, the protocol for prepare antibody coated membrane (4 pieces) was as follows. All antibody and buffer was warmed to room temperature prior to use.

\section{Carboxyl latex preparation:}

1. Pipette $50 \mu \mathrm{L}(40 \mathrm{mg} / \mathrm{mL}, 1.4 \mu \mathrm{m}$ diameter) latex microspheres and diluted with $150 \mu \mathrm{L}$ MES buffer.

2. Centrifuge the mixture to sediment the particles: $3000 \mathrm{rpm}$ for $5 \mathrm{~min}$.

3. Remove supernatant and re-disperse the pellet in $150 \mu \mathrm{L}$ MES buffer.

4. Centrifuge again and remove the supernatant from the particles.

5. Re-suspend pellet in $100 \mu \mathrm{L}$ MES buffer by vortexing to ensure a completely suspend of the microsphere particles.

\section{Preparation of antibody-labeled latex beads:}

1. Add 10 mg EDAC powder into the latex suspension prepared above, and react for 15 min with constant vortex to activate the latex beads.

2. Centrifuge the mixture to sediment the activated latex beads: $3000 \mathrm{rpm}$ for $5 \mathrm{~min}$.

3. Remove supernatant and re-disperse the pellet in $100 \mu \mathrm{L}$ MES buffer.

4. Centrifuge again and remove the supernatant from the particles.

5. Repeat step 3-4 twice more for a total of 3 washes.

6. Re-suspend activated latex beads in $50 \mu \mathrm{L}$ MES buffer by vortexing to ensure a completely.

7. Add antibody solution $(1 \mathrm{mg} / \mathrm{mL}, 250 \mu \mathrm{L}, 10 \mathrm{x}$ excess) to the latex suspension prepared above. For the antibody solutions that contain sodium azide, they were treated by centrifuge filters to remove the azide prior to binding the antibody to latex beads.

8. Incubate latex/antibody mixture with gentle mixing at room temperature for 5 hours.

9. Centrifuge to separate the antibody-labeled latex beads from unbound antibody solution.

10. Remove ad retain supernatant for the preparation of more antibody-labeled latex beads.

11. Re-suspend the beads in $100 \mu \mathrm{L}$ PBS buffer.

12. Centrifuge again to sediment the beads.

13. Repeat steps 11-12 twice more for a total of 3 washes.

14. Re-suspend the final latex beads in $100 \mu \mathrm{L}$ PBS buffer with $0.1 \%$ glycine. Store at $4^{\circ} \mathrm{C}$ until use.

Coat the antibody-labeled latex beads onto the glass-fiber membrane: Several 3-mm diameter glassfiber filter membranes (1.2 $\mu \mathrm{m}$ retention) were placed on an absorbent pad. Pipette re-suspend final latex bead prepared above onto the membranes, $25 \mu \mathrm{L}$ for each membrane. Antibody coated membranes were allow to dry and placed at the bottom of the antibody column. 


\section{S7. Sample preparation}

Standard proteins were first dissolved in water at a concentration $1 \mathrm{mg} / \mathrm{ml}$ and then diluted with spray solvent (methanol/water 50:50 v: v, with 2\% acetic acid) to the desired concentration. Experiments were performed on the same day that the protein samples were prepared. Human plasma was separated from donor human blood from K2EDTA treated vials, and stored at $-4^{\circ} \mathrm{C}$. ATTR samples were stored at $-20^{\circ} \mathrm{C}$ and brought to room temperature prior to use. All plasma samples were diluted 1:10 with PBS buffer. Purchased antibodies were purified using the centrifugal filter to remove the azide from the store buffer. All plasma and buffer was warmed to room temperature prior to use. 


\section{S8. Experimental methods}

Direct Spray Ionization Using Paper and PE Substrate. Detection limits of standard protein samples were obtained by direct spray ionization from paper of PE substrate. The size of the pentangle shaped substrate was

$\sim 5 \mathrm{~mm} \times 8 \mathrm{~mm}$ (base $\times$ height). Standard protein samples were diluted with 50:50 (v:v) methanol-water with $2 \%$ acetic acid to different concentrations. $20 \mu \mathrm{L}$ sample was deposited to the rear of the substrate and wicked through the substrate to the tip by capillarity. The paper or PE substrate, which was cut to a sharp point, was positioned $5 \mathrm{~mm}$ away from the atmospheric pressure inlet of the mass spectrometer and a high voltage of 5 $\mathrm{kV}$ was then applied to the spray substrate, inducing an electrospray at the tip of the substrate. The solvent evaporates from the charged droplets generated by the electrospray process, leaving gas phase ions of the analyte molecules, which can then be detected by a mass spectrometer.

Ionization Using Antibody Cartridge. As shown in the TOC figure, the diluted human plasma samples (1:10 dilution, $20 \mu \mathrm{L}$ plasma diluted with $180 \mu \mathrm{L}$ deionized water) were added to the antibody column. The sample wicked through the antibody column and subsequently onto the absorbent pad contained within the bottom part of the cartridge. As the sample passed through the antibody column (within $5 \mathrm{~min}$ ), the target proteins were retained on the antibody column while the excess matrix was absorbed onto the waste pad. The sample was then washed by applying $400 \mu \mathrm{L}$ of deionized water to the antibody column; the deionized water also wicked through the column onto the absorbent pad. The target proteins were recovered from the antibody column and analyzed by sliding the column holder (as well as the antibody column) over and pushing the antibody column down so that the antibody column was in contact with the pentagon-shaped CNT-PE substrate rather than the waste pad. The cartridge was placed in front of the inlet to the mass spectrometer, and the extraction/spray solvent (typically 1:1 methanol:water with $2 \%$ acetic acid) was added to the top of the enrichment column. The solvent wicked through the antibody column, recovering the proteins in the process, and onto the spray substrate passively. For the detection of plasma TTR, $25 \mathrm{mM}$ TECP-HCl was added to the spray solvent to perform the online reduction of the TTR posttranslational modifications. The reduction reaction was allowed to proceed on-cartridge for 5 minutes prior to ionization. Ionization was induced directly from the paper substrate by applying a high voltage ( $5 \mathrm{kV}$ typically) to the CNT-PE tip through a wire inserted from the side of the cartridge.

Mass Spectrometry, Data Collection, and Data Processing. All the experiments were carried out with a Q Exactive Focus mass spectrometer (Thermo Fisher Scientific, San Jose, CA, USA). The instrument parameters were as follows: Positive ion mode in all experiments; Spray voltage typically $5 \mathrm{kV}$, spray voltage may vary in different cases to make the spray current at $\sim 0.2 \mu \mathrm{A}$; Resolution 70,000; AGC target 1e6; Maximum inject time $100 \mathrm{~ms}$; Capillary temperature $320^{\circ} \mathrm{C}$; S-lens RF level 60.0; In the detection of apolipoprotein, hemoglobin, and transthyretin from diluted human plasma sample, in-source CID was set at $25.0 \mathrm{eV}$, Microscans set at 1 for full MS scan and set at 5 for SIM scan, SIM scan window 7.0 Da for apolipoprotein and 1.2 Da for hemoglobin. The software MagTran was used to generate the deconvoluted MS spectra of human plasma transthyretin. 
S9. Taylor cone formed from CNT-coated paper

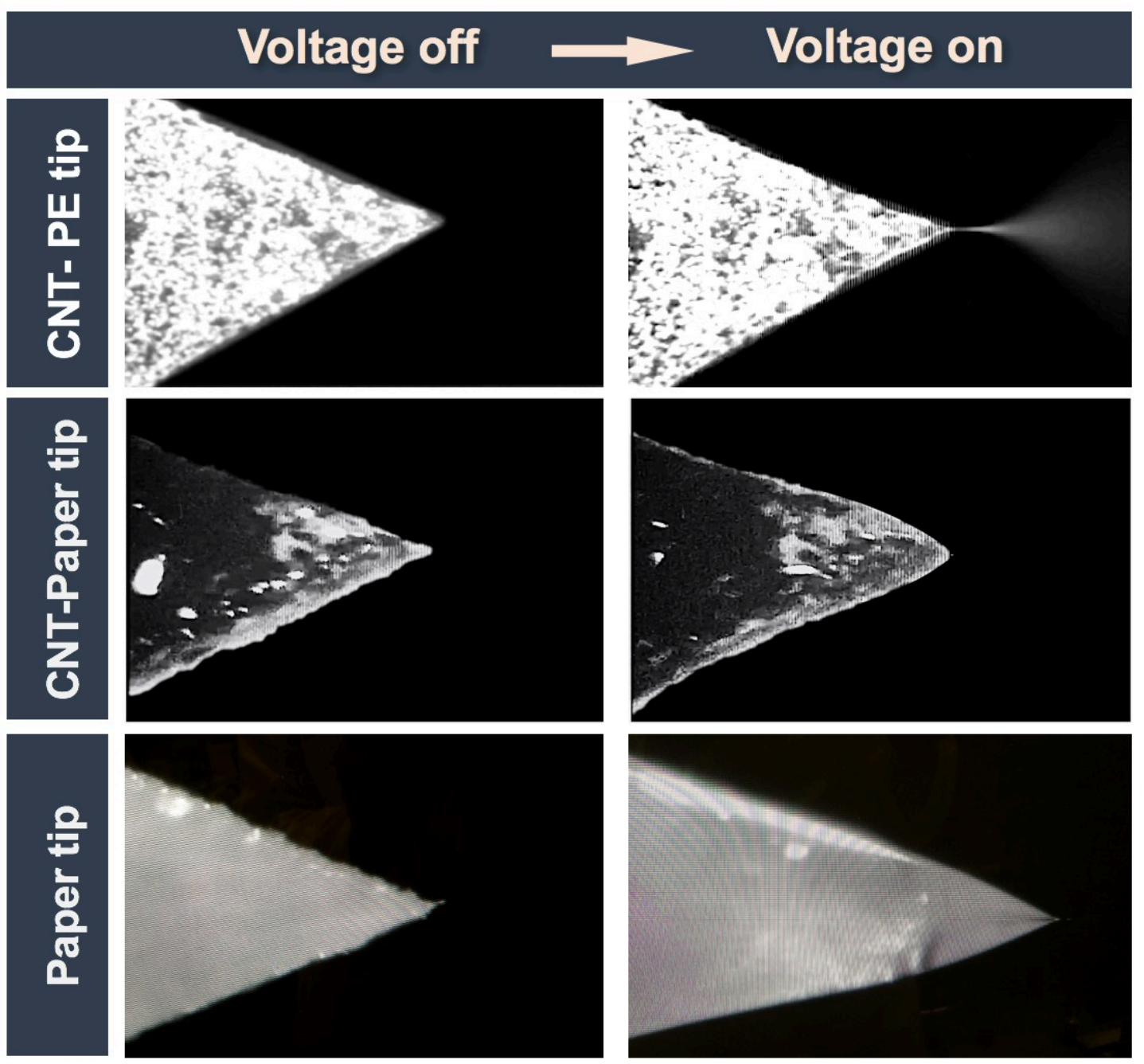

Figure S3. Pictures of three different substrates before (left) and after (right) application of high voltage. The CNT-PE (top row) and Paper (bottom row) are reproduced from the main text for comparison to CNT-coated paper (middle row). 


\section{S10. Reduction of TTR posttranslational modifications}

The reduction of TTR posttranslational modifications was performed during the protein extraction step. The TCEP-HCl was spiked into the spray solvent (methanol: water $1: 1 \mathrm{v}$ : v, with $2 \%$ acetic acid) at a concentration $25 \mathrm{mM}$. As shown in Figure S3, some high relative abundance posttranslational modifications, such as SCysteine and S-Glutathione were diminished after the TCEP-HCl reduction. The relative signal intensity of free thiol wild-type TTR increased significantly. However, the posttranslational modifications S-Sulfonation still exist in the spectrum. Increase the concentration of TCEP-HCl (from $25 \mathrm{mM}$ to $50 \mathrm{mM}$ ) or reduction time (from $5 \mathrm{~min}$ to $20 \mathrm{~min}$ ) can not remove the S-Sulfonation TTR from the sample. The reduction time was then optimized at $5 \mathrm{~min}$ with $25 \mathrm{mM}$ TCEP-HCl in spray solvent. The reduction greatly simplifies the data analysis and quantification of human plasma TTR, as well as the mutant.
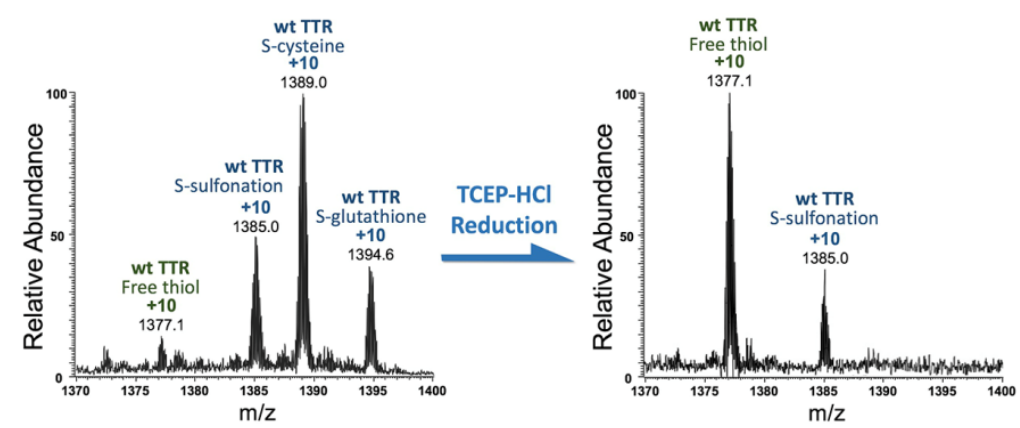

Figure S4. TCEP-HCl reduction significantly reduces the posttranslational modifications of plasma TTR and enhances the MS response of free thiol wild-type TTR. 


\section{S11. Protein retention by the spray substrates}

An $80 \mu \mathrm{L}$ aliquot of a $3 \mu \mathrm{g} / \mathrm{mL}$ of cytochrome $\mathrm{C}$ from equine heart was dissolved in the spray solvent and passed through either the paper spray substrate or the CNT-PE spray substrate in a manner consistent with the typical analysis. A $50 \mu \mathrm{L}$ aliquot was recovered from each spray substrate, and it was spiked with $0.5 \mu \mathrm{g}$ of an internal standard protein (cytochrome c from Saccharomyces cerevisiae). The recovery of two substrates can be compared using the following formula:

$$
\frac{R_{C N T-P E}}{R_{\text {paper }}}=\frac{A U C_{A, C N T-P E}}{A U C_{A, p a p e r}} / \frac{A U C_{L, C N T-P E}}{A U C_{L, p a p e r}}
$$

Where $\frac{\boldsymbol{R}_{\boldsymbol{C N T}-\boldsymbol{P E}}}{\boldsymbol{R}_{\text {paper }}}$ is the relative recovery between the two substrates and $A U C_{A}$ and $A U C_{L}$ are the areas under the curve for the analyte and internal standard, respectively. See Vega et al, Journal of the American Society of Mass Spectrometry 27(4), 726-734 (2016) for a full description of the data analysis.

In the case of cytochrome C, $\frac{\boldsymbol{R}_{\boldsymbol{C N T}-\boldsymbol{P E}}}{\boldsymbol{R}_{\text {paper }}}$ was $1.8 \pm 0.2(\mathrm{~N}=5)$, indicating that the protein recovered from the CNT-PE substrate was approximately double during the amount recovered from the paper substrate. This indicates that paper retained more target protein than CNT-PE. Because the limit of detection for cytochrome $\mathrm{C}$ was about 300X lower for the CNT-PE substrate compared to the paper, the lower retention by the CNT-PE is apparently a relatively minor contributor compared to the improvement in ionization efficiency. 


\section{S12. Recovery of captured protein}

To determine the elution efficiency from of the captured protein from the antibody column, antibody coated latex beads were incubated offline with $1.2 \mathrm{~mL}$ of a $10 \mu \mathrm{g} / \mathrm{mL}$ cytochrome c (equine heart) aqueous solution. The beads were then washed 3 times and divided into six equal aliquots. Three of them were coated to the membrane disc identically to the typical analysis. The other three were extracted offline using methanol: water 1:1 (v: v) with 2\% acetic acid for 10 minutes while vortexing. For both samples, an internal standard (cytochrome $\mathrm{C}$ from Saccharomyces cerevisiae, $0.6 \mu \mathrm{g}$ ) was spiked into the elution/spray solvent.

The direct elution method used for the cartridge analysis was compared to offline recovery using equation 1 above. $\frac{\boldsymbol{R}_{\text {direct }}}{\boldsymbol{R}_{\text {offline }}}$ was found to be $0.94(\mathrm{~N}=3)$, indicating that extended offline extraction did not elute anymore protein than the direct elution method used in the protein cartridge.

In a second experiment, the latex bead coated membranes were removed from the cartridge after analysis and subjected to offline extraction as described above. No protein was detectable in the offline extraction. This indicates that virtually all of the protein was eluted from the beads during the direct elution. 